\title{
Detection of nearly periodic spin period reversals in Vela $X-1$ on long time-scales: inkling of solar-like cycle in the donor star?
}

\author{
Amar Deo Chandra, ${ }^{1,2 \star}$ Jayashree Roy, ${ }^{3,1}$ P. C. Agrawal ${ }^{1,4}$ and Manojendu Choudhury ${ }^{5,1}$ \\ ${ }^{1}$ UM-DAE Centre for Excellence in Basic Sciences, University of Mumbai, Vidyanagari Campus, Kalina, Santacruz (East), Mumbai, Maharashtra 400098, India \\ ${ }^{2}$ Center of Excellence in Space Sciences India, Indian Institute of Science Education and Research Kolkata, Mohanpur 741246, West Bengal, India \\ ${ }^{3}$ Inter-University Center for Astronomy and Astrophysics, Post Bag 4, Pune, Maharashtra 411007, India \\ ${ }^{4}$ Senior Professor (Retd.), Dept. of Astronomy and Astrophysics, Tata Institute of Fundamental Research, Homi Bhabha Road, Mumbai 40005, India \\ ${ }^{5}$ Department of Physics, St. Xavier's College (Autonomous), 5 Mahapalika Marg, Mumbai 400001, Maharashtra, India
}

Accepted XXX. Received YYY; in original form ZZZ

\begin{abstract}
We explore the long-term evolution of the spin period of the High Mass X-ray Binary (HMXB) pulsar Vela X-1 over a period of $46 \mathrm{yr}$. Our analysis indicates nearly periodic variations in the spin period of the pulsar on time-scales of about $5.9 \mathrm{yr}$. There is suggestion of an overall spin-down behaviour of the pulsar though it is noticed that the source appears to stay near its equilibrium period $283.4 \mathrm{~s}$ since MJD 51000, with rather erratic spin-up/spin-down episodes around this value. Our study suggests nearly cyclic turnover in the spin behaviour of the pulsar from spin-up to spin-down regimes on time-scales of about 17-19 yr. To our knowledge this is the first report of periodic variation in the spin behaviour of a wind-fed accreting pulsar. We also observe erratic episodes of spin-up and spin-down behaviour on relatively shorter time-scales which is a well known archetype of this wind-fed X-ray pulsar. We investigate whether nearly periodic long-term spin period changes in the pulsar can be explained by using known mechanisms of torque reversals in the accretion powered neutron stars. It appears that changes in the accretion environment of the pulsar using current ideas can probably lead to long-term spin period changes in this X-ray pulsar.
\end{abstract}

Key words: stars: neutron - pulsars: individual: Vela X-1 - X-rays: binaries

\section{INTRODUCTION}

Vela X-1 (4U 0900-40) is an eclipsing High mass X-ray binary (HMXB) discovered during rocket borne X-ray observations in 1967 (Chodil et al. 1967). It is located at a distance of $\sim 2.0 \mathrm{kpc}$ (Sadakane et al. 1985; Nagase 1989) in the Vela constellation. Recent estimates using Gaia data infer distance of $2.42_{-0.17}^{+0.19} \mathrm{kpc}$ (Bailer-Jones et al. 2018). The system consists of a massive B0.5Ib supergiant HD 77581 (Hiltner et al. 1972; Brucato \& Kristian 1972; Vidal et al. 1973; Jones \& Liller 1973) having mass of about $\sim 23 \mathrm{M}_{\odot}$ and radius of $\sim 34 \mathrm{R}_{\odot}$ (Van Paradijs et al. 1976; Joss \& Rappaport 1984; Nagase 1989; van Kerkwijk et al. 1995) and a neutron star with mass $\sim 1.8 \mathrm{M}_{\odot}$ (Van Paradijs et al. 1976; Nagase 1989; Barziv et al. 2001; Rawls et al. 2011). The orbital period of the binary system is about nine days (Hiltner et al. 1972; Forman et al. 1973; Vidal et al. 1973; Watson \& Griffiths 1977; van Kerkwijk et al. 1995). Due to the close proximity of about $1.7 \mathrm{R}_{\star}$ (Conti 1978; Quaintrell et al. 2003) between the neutron star and its companion, the neutron star is immersed in the dense stellar wind of the donor star having typical mass loss rate of

^ E-mail: amar.deo.chandra@gmail.com about $\dot{M} \sim 10^{-6} \mathrm{M}_{\odot} \mathrm{yr}^{-1}$ (Hutchings 1974; Dupree et al. 1980; Nagase et al. 1986; Sako et al. 1999). A fraction of the stellar wind is captured and channelled along the strong magnetic field $\left(\sim 2.7 \times 10^{12} \mathrm{G}\right.$; (Kretschmar et al. 1996; Kreykenbohm et al. 2002; Coburn et al. 2002) of the neutron star onto the magnetic poles, producing regular X-ray pulsations caused by the spin period 283 s (Rappaport \& McClintock 1975; McClintock et al. 1976) of the neutron star. Although Vela X-1 is known to be a persistent source having luminosity of about $4 \times 10^{36} \mathrm{erg} \mathrm{s}^{-1}$ (McCray et al. 1984; Sadakane et al. 1985; Nagase et al. 1986; Kreykenbohm et al. 2002), it shows a plethora of X-ray variabilities such as sudden flares lasting a few minutes to several hours wherein the luminosity increases by several folds within very short time-scales of a few tens of seconds (Lapshov et al. 1992; Staubert et al. 2004; Kreykenbohm et al. 2008). Occurrence of sudden flares in this system are not so well understood and is believed to be due to enhanced accretion rate due to variabilities in the stellar wind from the companion star (Nagase et al. 1983; Haberl \& White 1990) or accretion of clumpy stellar wind (Staubert et al. 2004; Ducci et al. 2009; Fürst et al. 2010; Odaka et al. 2013). Some studies suggest that sudden flares might be related to formation of transient accretion disc (Inoue et al. 1984; Taam \& Fryxell 
1989; Haberl \& White 1990; Kreykenbohm et al. 2008). Another bizzare manifestation seen in Vela X-1 is occurrence of abrupt "off-states" wherein X-ray pulsations cessation (within less than the pulse period) is observed for several tens of minutes at a time (Inoue et al. 1984; Lapshov et al. 1992; Kreykenbohm et al. 1999, 2008; Doroshenko et al. 2011; Sidoli et al. 2015). These states are poorly understood and might be caused by changes in the accretion rate due to variabilities in the stellar wind (Lapshov et al. 1992; Coburn et al. 2002). Some earlier studies also suggest that "off-states" might be associated with formation of transient accretion discs (Inoue et al. 1984) or the accretion is choked due to the sudden onset of propeller effect (Kreykenbohm et al. 2008). It has also been suggested that the onset of these "off-states" can be caused due to transition from the higher luminosity Compton cooling regime to the lower luminosity radiative cooling regime (Shakura et al. 2013) or due to unstable hydrodynamic flows in the vicinity of the neutron star (Manousakis \& Walter 2015a). Recent numerical studies suggest formation of temporary accretion discs in wind-fed X-ray pulsars (El Mellah et al. 2019b,a; Karino et al. 2019) but conclusive evidence of their existence has been elusive. Interestingly, Liao et al. (2020) infer presence of temporary accretion disc in Vela X-1 during an extended low state lasting at least $30 \mathrm{ks}$ which was accompanied by unusual spin-up event and similar $\mathrm{Fe} \mathrm{K} \alpha$ fluxes compared to the preceding flaring period.

Long-term monitoring of the spin period of Vela X-1 since 1975 has shown erratic spin period variations over time which is an archetype of wind-fed X-ray pulsars (Deeter et al. 1989; Bildsten et al. 1997). Spin excursions on short time-scales are considered to be caused by the dynamic accretion torque acting on the neutron star as a result of changes in the stellar wind from the donor star (Kreykenbohm et al. 2008). Vela X-1 is known to show variable episodes of spin-up and spin-down (Nagase et al. 1981). The evolution of spin period is most appropriately described by a random walk model (Tsunemi et al. 1989; Ziolkowski 1985). Although the source shows strong pulse to pulse variations which is a tell-tale manifestation of fluctuating rate of accretion (Staubert et al. 1980; Nagase et al. 1984b; Kretschmar et al. 1997), pulse profiles folded over several pulse periods show remarkable stability (for 10 pulses or more; Staubert et al. (1980), even over decades (Raubenheimer 1990)). This suggests that the magnetic environment of the neutron star is very stable on long time-scales. Recent measurements of surface magnetic fields of X-ray pulsars using detection of cyclotron resonance scattering effects suggest that the magnetic field of neutron stars are almost constant on very long time-scales of about $10^{8} \mathrm{yr}$ (Makishima et al. 1999). However, recent studies have found changes in cyclotron line energies in Vela X-1 on long time-scales using the Neil Gehrels Swift Observatory/BAT observations (La Parola et al. 2016; Ji et al. 2019). This suggests that the inferred magnetic field decays at the rate of about $3 \times 10^{10} \mathrm{G} \mathrm{yr}^{-1}$ (La Parola et al. 2016). The most recent spin evolution study of Vela X-1 found the pulsar showing spin-down behaviour (Kreykenbohm et al. 1999).

Investigations of spin period variations on various time-scales (few tens of days to several hundred days) have been carried out in several X-ray pulsars and at least one torque reversal has been detected in some of these pulsars (Cen X-3 (Tsunemi 1989); several pulsars studied by Nagase (1989) and references therein; OAO 1657-415 (Chakrabarty et al. 1993); several pulsars monitored and studied by the Burst and Transient Source Experiment (BATSE) (Bildsten et al. 1997); 4U 1626-67 Wilson et al. (1993), Chakrabarty et al. (1997a), Camero-Arranz et al. (2009); GX 1+4 Makishima et al. (1988), Chakrabarty et al. (1997b), González-Galán et al. (2012); 4U 1907+09 Fritz et al. (2006), Inam et al. (2009); 4U 0114+650 (Hu et al. 2016); LMC X-4 (Molkov et al. 2016); NGC 300 ULX1 (Vasilopoulos et al. 2019); 2S 1845-024 and several other pulsars monitored and studied by the Gamma-ray Burst Monitor (GBM) aboard the Fermi Gamma-ray Space Telescope (Malacaria et al. 2020)). A long-term periodicity of about $9 \mathrm{yr}$ and $6.8 \mathrm{yr}$ has been detected in the spin evolution of Cen X-3 (Tsunemi 1989) and LMC X-4 (Molkov et al. 2016) respectively. It is intriguing to note that GX $1+4$, which is a disc-fed pulsar, has shown only one transition from secular spin-up to monotonic spin-down behaviour (Makishima et al. 1988; Chakrabarty et al. 1997b; González-Galán et al. 2012) during its spin evolution monitored over almost five decades. Cen X-3 has a massive O6-8 III type companion star V779 Cen (Krzeminski 1974) which has a strong wind. However, presence of an accretion disc has been detected in this system (Tjemkes et al. 1986) which suggests that accretion induced spin changes in this pulsar are mainly driven by accretion from the disc. This makes Cen X-3 a predominantly disc-fed pulsar. The companion star of LMC X-4 is an O8 III type massive star (Kelley et al. 1983; Falanga et al. 2015) and the neutron star accretes from an accretion disc in this system (Lang et al. 1981) making this a disc-fed source. It should be noted that though Cen X-3 and LMC X-4 are disc-fed pulsars unlike Vela $\mathrm{X}-1$ which is a wind-fed pulsar, they also exhibit long-term periodicity in their spin evolution. This makes Vela X-1 to be the first wind-fed X-ray pulsar where such a long-term periodicity (on time-scales of years) in the spin evolution has been detected.

In this paper, we investigate the long-term ( $~ 46$ yr) spin evolution of Vela X-1 using spin period measurements from 24 different observatories. Most of the spin period measurements are taken from literature and we deduce spin periods, not reported earlier, from using the Rossi X-ray Timing Explorer (RXTE)/Proportional Counter Array (PCA) and the AstroSat/Large Area X-ray Proportional Counter (LAXPC) observations. The complete list of spin period measurements is shown in the appendix 5. The paper is organized as follows. After the introduction, in section 2 we first describe observations and data analysis from archival RXTE/PCA observations of Vela X-1. Then we describe recent observations from the AstroSat mission and LAXPC data analysis procedures. In section 3 we describe our main results related to the detection of long-term spin-down trend in Vela X-1 and detection of nearly periodic spin period reversals on long time-scales. In section 4 we discuss possible changes in the accretion landscape of this wind-fed $\mathrm{X}$-ray pulsar using current ideas which can explain nearly periodic long-term torque reversals. A summary of all our findings is presented in section 5 .

\section{OBSERVATIONS AND DATA ANALYSIS}

The main goal of our work is to explore the long-term spin period evolution of Vela X-1 since its discovery almost five decades ago. To the best of our knowledge, such a long-term ( $46 \mathrm{yr})$ spin evolution study of a wind-fed X-ray pulsar is being reported for the first time. It should be noted that the spin period evolution over periods of $\sim 10$ to $20 \mathrm{yr}$ has been studied earlier in Cen X-3 (Tsunemi 1989) and LMC X-4 (Molkov et al. 2016) which are both disc-fed pulsars. We use all the spin period measurements reported in literature and infer new spin periods using archival data from the 
Rossi X-ray Timing Explorer mission (RXTE, Bradt et al. (1993) and the currently operational AstroSat mission (Agrawal 2006).

\subsection{RXTE observations and data reduction}

The Rossi X-ray Timing Explorer (RXTE) was operational for about 16 yr from 1996 February until 2012 January. During its lifetime it observed Vela X-1 numerous times and about a hundred archival RXTE observations are available for this star, with each observation ranging from about a thousand seconds to $\sim 40 \mathrm{ks}$. We have analysed some of these archival observations to obtain the spin period of Vela X-1 at different epochs. The log of RXTE observations used in our study are shown in Table 1 . Three science instruments were flown onboard this mission viz. the Proportional Counter Array (PCA, Jahoda et al. (1996)), the High Energy Timing Experiment (HEXTE; Rothschild et al. (1998)) and the All Sky Monitor (ASM; Levine et al. (1996)). In our present work, we have used archival data from the PCA instrument. The PCA consists of five co-aligned Xenon proportional counter units (PCUs) with total effective area of about $6500 \mathrm{~cm}^{2}$ and sensitive in the energy range from $2 \mathrm{keV}$ to $\sim 60 \mathrm{keV}$ (Jahoda et al. 1996). We used data only from the PCU2 as it was functional most of the time during the RXTE lifespan, while other PCUs suffered occasional breakdowns. We analysed the RXTE data using the standard NASA HEASOFT software package (version 6.12) released by the High Energy Astrophysics Science Archive Research (HEASARC) Center. ${ }^{1}$ The detailed procedure of data analysis using FTOOLS are illustrated in "The RXTE Cook Book: Recipes for Data Analysis and Reduction "2 and "The ABC of XTE" 3 , written and maintained by RXTE Guest Observer Facility (RXTE GOF).

The X-ray light curves with $125 \mathrm{~ms}$ resolution were extracted using standard RXTE analysis software FTOOLS ver. 6.13. All the light curves were rebinned in $1.0 \mathrm{~s}$ bins before further processing. When determining the source "goodtime" intervals, the following data selection criteria were used for the extraction of source counts. To avoid possible contamination due to X-rays from the Earth's limb, data was extracted only when the satellite was pointing more than $10^{\circ}$ above the horizon. To avoid possible contamination from activation in the detectors due to the high particle rates in the SAA (South Atlantic Anomaly) passages, data were rejected from a 40 min interval beginning with the satellite entering the SAA. It was also ensured that the satellite pointed within $0.02^{\circ}$ of the source position. Some of the observations had low exposure time $(\sim 3000 \mathrm{~s})$ and so they were merged with other close observations to generate a light curve with long exposure. In order to remove the effect caused by the motion of the satellite and the Earth, the observed times of arrival of the pulse were converted to those of the barycentre of the solar system. The barycentric corrections to the event data files were applied using the FTOOLS utility 'FAXBARY'. The light curves were rebinned to $16 \mathrm{~s}$ to enhance the signal-to-noise ratio (SNR) before periodicity searches. The resulting light curve has data gaps in between and so we used the method of Scargle (Scargle 1982) for periodicity searches in RXTE data. The derived pulse periods are tabulated in the appendix 5.

1 http://heasarc.gsfc.nasa.gov/

2 https://heasarc.gsfc.nasa.gov/docs/xte/recipes/cook_ book.html

${ }^{3}$ http://heasarc.gsfc.nasa.gov/docs/xte/abc/contents.html

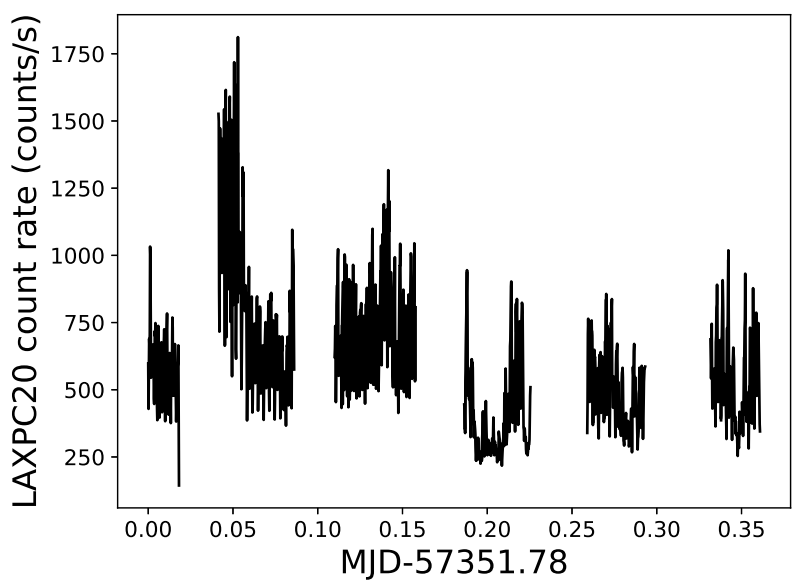

Figure 1. Light curve of Vela X-1 in the 3-80 keV energy band using AstroSat data from orbit $869-874$. The light curve has been rebinned to $16 \mathrm{~s}$. The gaps in the light curve are due to the passage of the satellite through the South Atlantic Anomaly regions.

\subsection{LAXPC observations and data reduction}

We have analysed observations from the Large Area X-ray Proportional Counter (LAXPC) instrument onboard the AstroSat mission (Agrawal 2006). The AstroSat/LAXPC observations were carried out on 2015 November 25 and 2015 November 26 covering orbits 869-874, during the Performance Verification (PV) phase of AstroSat. We have also analysed the Target of Opportunity (ToO) observations of Vela X-1 from 2019 March 12 to 2019 March 16 covering 13 orbits available on the ISRO Science Data Archive for the AstroSat mission ${ }^{4}$. The log of AstroSat observations used in our study is shown in Table 2.

LAXPC consists of 3 identical collimated detectors (LAXPC10, LAXPC20 and LAXPC30), having 5 anode layer geometry with $15 \mathrm{~cm}$ deep X-ray detection volume providing an effective area of about $4500 \mathrm{~cm}^{2}$ at $5 \mathrm{keV}, 6000 \mathrm{~cm}^{2}$ at $10 \mathrm{keV}$ and about $5600 \mathrm{~cm}^{2}$ at about $40 \mathrm{keV}$ (Roy et al. 2019; Chandra et al. 2020). The arrival times of X-ray photons are recorded with a time resolution of $10 \mu \mathrm{s}$. The details of the characteristics of the LAXPC instrument are available in (Yadav et al. 2016; Agrawal et al. 2017; Roy et al. 2016). The calibration details of LAXPC instrument are given in Antia et al. (2017). We have used softwares available from the AstroSat Science Support Cell ${ }^{5}$ to reduce Level-1 data to Level2 data. Level-2 data contains (i) light curve in broad band counting mode (modeBB) and (ii) event mode data (modeEA) with information about arrival time, pulse height and layer of origin of each detected X-ray and (iii) housekeeping data and parameter files are stored in mkf file. We have used laxpc software tool having single routine to extract spectra, light curve and background, "LAXPCSOFT " to extract light curves having $1 \mathrm{~s}$ resolution using the event mode data. Fig. 1 shows light curve of Vela X-1 in the 3-80 keV energy band obtained using LAXPC20 data from orbit 869-874.

We correct X-ray photons arrival times to the solar system barycentre using the AstroSat barycentric correction utility

\footnotetext{
4 https://www.issdc.gov.in/astro.html

5 http://astrosat-ssc.iucaa.in/?q=laxpcData
} 


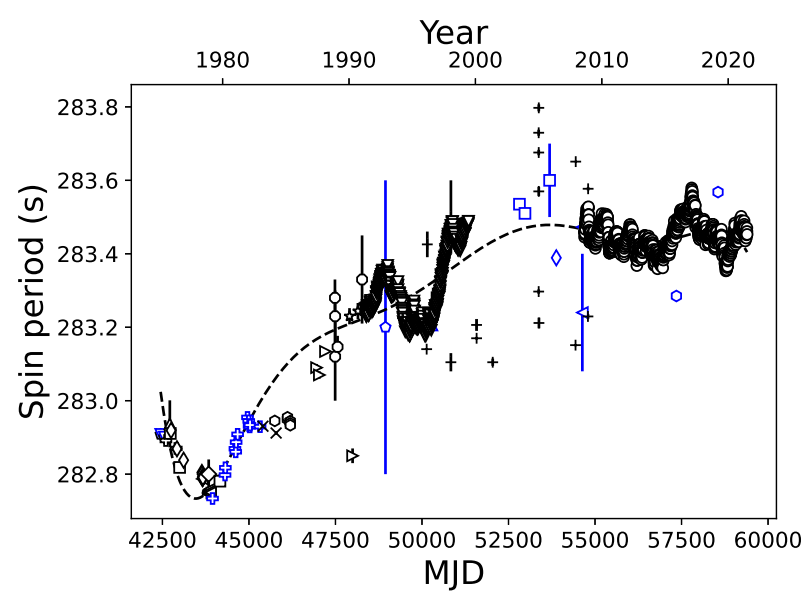

Figure 2. Long-term spin history of Vela X-1 from 1975 February until 2021 June. The general trend of secular spin-down of the X-ray pulsar superposed on random episodes of spin-up and spin-down behaviour is discernible. The dashed curve shows the best fit polynomial to the spin evolution of Vela X-1. A careful look at the long-term pulse period evolution also suggests presence of cyclic trend in the long-term spin evolution of Vela X-1. The different markers indicate spin period measurements from different observatories $(\square$ :

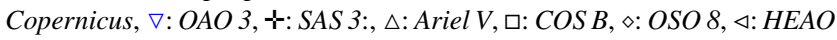
$1, \diamond:$ AIT/MPI balloon X-ray detector, †: Hakucho, x: Tenma, $\bigcirc:$ EXOSAT, $\triangleright:$ Ginga, $\bigcirc:$ KVANT, *: GRANAT, $\bigcirc:$ ROSAT, $\nabla:$ CGRO/BATSE, +: RXTE, $\triangle:$ BeppoSAX, $\square:$ INTEGRAL, $\diamond: X M M-N e w t o n, \triangleleft:$ Suzaku, X: NuSTAR, $\bigcirc:$ Fermi/GBM, $\bigcirc$ : AstroSat). The symbols are shown in different colours for the various missions. The dark shaded patches are due to close cadence of BATSE and Fermi observations such that they almost overlap showing dark patches.

'aslbary'. The orbit files for barycentric correction are generated using AstroSat orbit file generator ${ }^{6}$. 'as lbary' requires HEASOFT software package (version 6.17 or higher) and so we have used the latest HEASOFT software package (version 6.26) for our analysis. The light curves were rebinned to $16 \mathrm{~s}$ to enhance the signal-to-noise ratio (SNR) before searches for periodicity. We use the FTOOLS subroutine efsearch to obtain the best estimated pulse periods. The inferred pulse periods are shown in the appendix 5.

\section{RESULTS}

\subsection{Long-term spin-down apparition in Vela X-1}

Making use of all the spin periods of Vela X-1 reported in literature as well as the values derived from our analysis of RXTE/PCA and AstroSat/LAXPC, we construct the long-term spin history of Vela $\mathrm{X}-1$ which is shown in Fig. 2.

The spin history spans a period of 46 yr (1975 February until 2021 June) and this is probably the longest time period over which the spin period for an accretion powered pulsar has been measured. So far, long-term trends in spin period have been observed only in Xray pulsars harbouring an accretion disc (Tsunemi 1989; GonzálezGalán et al. 2012; Molkov et al. 2016; Vasilopoulos et al. 2019). The remarkable long-term secular spin-down trend of Vela X-1 is clearly discernible, albeit it shows random alterations between

\footnotetext{
${ }^{6}$ http://astrosat-ssc.iucaa.in: 8080/orbitgen/
}

spin-up and spin-down regimes on shorter time-scales, which is the paradigm of wind-fed pulsars (Deeter et al. 1989; Bildsten et al. 1997). Vela X-1 was found to be on a spin-up evolutionary track after its discovery in 1975. This trend continued for four years until 1979 and then Vela X-1 experienced an abrupt transition to spindown regime, which lasted about three years. It is intriguing to note that the culmination of this spin-down phase has spin period value comparable to that when its spin period was first measured in 1975. Vela X-1 continued its spin adventure and thereafter switched into a bizzare mode, wherein the pulse period was more or less stable on time-scale of about 3-4 yr. This almost constant spin period phase is really intriguing for a wind-fed pulsar as wind-fed accretion powered pulsars are known to show random variations in spin period on short time-scales (as short as within a few days, Boynton et al. (1984, 1986); Deeter et al. (1989); Baykal \& Oegelman (1993); de Kool \& Anzer (1993)). Thereafter, the pulsar switched to a remarkable longterm spin-down behaviour (with superposed sporadic episodes of spin-up and spin-down behaviour on relatively shorter time-scales) lasting almost three decades or so. A careful examination of the Fig. 2 suggests that the rate of spin-up and spin-down can vary significantly on various time-scales during the entire recorded spin history of Vela X-1. We observe dips in spin period around MJD 44000, 50000, 52000, 54000, 57000 and 59000 which hints that there might be cyclic transitions between spin-up and spin-down on time-scales of about a few thousand days or so. Fig. 3 shows the spin evolution of Vela X-1 for about $46 \mathrm{yr}$ and we identify three distinct episodes of torque reversals from spin-down to spinup regimes around MJD 44000, 50000 and 57000. These torque reversals occur nearly in a cyclic manner after about 6000-7000 d ( 16.5-19.4 yr) which is really intriguing for a wind-fed pulsar which are known to show random torque reversals on much smaller time-scales. Interestingly, the radius of curvatures of the parabolas fitted to the spin evolution around these epochs are different and asymmetric. This suggests that the pulsar spends different durations in these states and the rate of spin-up and spin-down are markedly different during these torque reversals.

\subsection{Nearly periodic spin period reversals in Vela X-1 on long time-scales}

We further probe possible cyclic variations in the spin period of Vela $\mathrm{X}-1$ by constructing the long-term averaged spin history using a smoothing window of $300 \mathrm{~d}$ (Fig. 4).

This averages out random transitions in spin period on short time-scales and clearly brings out the nearly cyclic variations in spin period on long time-scales. We observe prominent dips in the spin period around MJD 44000, 48000, 50000, 52000, 54000, 57000 and 59000. It is really intriguing that the long-term averaged spin variations in Vela $\mathrm{X}-1$ is almost cyclic having periodicity of about $2000 \mathrm{~d}$. The tiny dip in spin period around MJD 46000 is likely an artefact of smoothing. Besides, the rate of spin-up and spin-down varies significantly and quite differently during each of these slow transitions in spin period as is evident from the asymmetric nature of these gradual spin period changes occurring on long time-scales. These subtle observations gleaned from averaged spin history in Vela X-1 can be helpful in probing the underlying phenomena causing nearly cyclic spin changes in this pulsar on time-scale of about $2000 \mathrm{~d}$. In case the spin-down rate is smaller than the spin-up rate during these gradual transitions (e.g. notice transitions in spin period around MJD 48000, 50000 and 52000), it may suggest a phenomena involving some kind of relaxation effect such as a complex interplay of slow changes in the accretion rate 
Table 1. Log of RXTE PCU2 observations used in this study.

\begin{tabular}{|c|c|c|c|c|c|}
\hline S. no. & ObsID & Time of Observation & Exposure (s) & MJD (mid) & Useful exposure (s) \\
\hline 1 & 10141-01-02-00 & 1996-02-22 02:15:14.7 & 6567 & 50135.16 & 5952 \\
\hline 2 & $10144-02-01-00$ & 1996-03-11 15:36:59.6 & 9956 & 50153.74 & 9232 \\
\hline 3 & $10142-01-01-00$ & 1996-07-27 16:31:40.2 & 31953 & 50291.86 & 16176 \\
\hline 4 & $30102-01-01-00$ & 1998-01-21 19:20:41 & 40833 & 50834.98 & 15744 \\
\hline \multirow[t]{3}{*}{5} & 40073-01-01-03 & 2000-02-03 05:04:25.8 & 2167 & 51577.30 & 5936 \\
\hline & 40073-01-01-02 & 2000-02-03 06:34:33.6 & 2212 & & \\
\hline & 40073-01-01-01 & 2000-02-03 08:13:07.7 & 2203 & & \\
\hline \multirow[t]{6}{*}{6} & 40073-01-01-04 & 2000-02-03 22:37:04.2 & 3951 & 51578.33 & 27632 \\
\hline & 40073-01-02-00 & 2000-02-04 00:00:06.9 & 10677 & & \\
\hline & 40073-01-02-01 & 2000-02-04 05:10:02.8 & 1373 & & \\
\hline & 40073-01-02-02 & 2000-02-04 06:36:04.3 & 1971 & & \\
\hline & 40073-01-02-03 & 2000-02-04 08:12:04.6 & 2090 & & \\
\hline & 40073-01-02-04 & 2000-02-04 09:48:04.9 & 16490 & & \\
\hline \multirow[t]{2}{*}{7} & 40073-01-03-00 & 2001-05-14 20:03:16.7 & 14154 & 52044.12 & 23616 \\
\hline & 40073-01-03-01 & 2001-05-15 03:47:05.3 & 12225 & & \\
\hline \multirow[t]{4}{*}{8} & 90083-01-01-05 & 2005-01-01 00:08:48.8 & 1938 & 53371.22 & 18880 \\
\hline & 90083-01-01-06 & 2005-01-01 01:20:03.8 & 2149 & & \\
\hline & 90083-01-01-07 & 2005-01-01 02:51:00.9 & 13735 & & \\
\hline & 90083-01-01-10 & 2005-01-01 09:08:58.3 & 3779 & & \\
\hline \multirow[t]{5}{*}{9} & 90083-01-01-08 & 2005-01-02 02:52:52.3 & 13330 & 53372.50 & 32560 \\
\hline & 90083-01-01-12 & 2005-01-02 08:44:59.7 & 4000 & & \\
\hline & 90083-01-01-04 & 2005-01-02 10:20:00.4 & 17275 & & \\
\hline & 90083-01-01-13 & 2005-01-02 18:27:57.9 & 2217 & & \\
\hline & 90083-01-01-14 & 2005-01-02 20:05:00.4 & 2395 & & \\
\hline \multirow[t]{10}{*}{10} & 90083-01-01-15 & 2005-01-03 00:54:19 & 2237 & 53373.52 & 33712 \\
\hline & $90083-01-01-16$ & 2005-01-03 02:30:29.7 & 2396 & & \\
\hline & $90083-01-01-25$ & 2005-01-03 03:47:29.5 & 3477 & & \\
\hline & 90083-01-01-27 & 2005-01-03 05:22:29.3 & 3417 & & \\
\hline & $90083-01-01-28$ & 2005-01-03 06:51:29.7 & 3777 & & \\
\hline & 90083-01-01-17 & 2005-01-03 08:26:29.5 & 2997 & & \\
\hline & 90083-01-01-02 & 2005-01-03 09:56:00.1 & 17155 & & \\
\hline & 90083-01-01-29 & 2005-01-03 18:03:00.6 & 2573 & & \\
\hline & 90083-01-01-18 & 2005-01-03 19:41:01 & 2514 & & \\
\hline & 90083-01-01-19 & 2005-01-03 22:55:38.8 & 1777 & & \\
\hline \multirow[t]{5}{*}{11} & 90083-01-01-20 & 2005-01-04 01:59:05.3 & 2504 & 53374.54 & 30192 \\
\hline & $90083-01-01-21$ & 2005-01-04 03:18:00.9 & 7195 & & \\
\hline & 90083-01-01-30 & 2005-01-04 06:24:01.2 & 16000 & & \\
\hline & 90083-01-01-03 & 2005-01-04 11:17:01 & 20034 & & \\
\hline & $90083-01-01-22$ & 2005-01-04 22:30:52.7 & 3000 & & \\
\hline \multirow[t]{3}{*}{12} & $90083-01-01-23$ & 2005-01-05 01:29:52.2 & 14531 & 53375.54 & 26160 \\
\hline & 90083-01-01-24 & 2005-01-05 07:35:03.3 & 4000 & & \\
\hline & 90083-01-01-01 & 2005-01-05 09:11:01 & 14397 & & \\
\hline 13 & 90083-01-02-01 & 2005-01-09 05:18:41.2 & 8195 & 53379.29 & 6896 \\
\hline \multirow[t]{5}{*}{14} & 93039-02-01-08 & 2007-12-03 02:56:10.2 & 995 & 54437.39 & 33600 \\
\hline & $93039-02-01-03$ & 2007-12-03 04:12:18.1 & 2179 & & \\
\hline & 93039-02-01-10 & 2007-12-03 05:15:33.7 & 16009 & & \\
\hline & 93039-02-01-09 & 2007-12-03 11:34:03.1 & 3833 & & \\
\hline & 93039-02-01-04 & 2007-12-03 13:16:18.3 & 17662 & & \\
\hline \multirow[t]{10}{*}{15} & 93039-01-01-00 & 2007-12-07 11:24:19.9 & 17361 & 54442.42 & 67264 \\
\hline & 93039-01-01-11 & 2007-12-07 19:53:23.1 & 8289 & & \\
\hline & 93039-01-01-06 & 2007-12-08 00:15:02.9 & 2989 & & \\
\hline & 93039-01-01-09 & 2007-12-08 01:49:19.5 & 3317 & & \\
\hline & 93039-01-01-04 & 2007-12-08 03:09:17.3 & 20425 & & \\
\hline & 93039-01-01-05 & 2007-12-08 11:31:03.4 & 952 & & \\
\hline & 93039-01-01-01 & 2007-12-08 12:43:19.8 & 23777 & & \\
\hline & 93039-01-01-07 & 2007-12-08 23:46:02.8 & 3049 & & \\
\hline & 93039-01-01-10 & 2007-12-09 01:21:19.9 & 3437 & & \\
\hline & 93039-01-01-02 & 2007-12-09 02:44:16.5 & 16883 & & \\
\hline \multirow[t]{4}{*}{16} & 93039-01-02-02 & 2008-11-25 04:22:05.7 & 18403 & 54795.52 & 29184 \\
\hline & 93039-01-02-08 & 2008-11-25 11:36:03.2 & 2451 & & \\
\hline & 93039-01-02-05 & 2008-11-25 13:15:13.5 & 5551 & & \\
\hline & 93039-01-02-03 & 2008-11-25 17:44:05.3 & 7430 & & \\
\hline
\end{tabular}


Table - continued Log of RXTE PCU2 observations used in this study.

\begin{tabular}{cccccc}
\hline \hline S. no. & ObsID & Time of Observation & Exposure (s) & MJD (mid) & Useful exposure (s) \\
\hline \multirow{2}{*}{17} & $93039-02-02-00$ & $2008-11-30$ 01:27:03.7 & 22072 & 54801.04 & 42480 \\
& $93039-02-02-01$ & $2008-11-3010: 55: 37.9$ & 2263 & & \\
& $93039-02-02-02$ & $2008-11-3014: 01: 03.6$ & 8676 & & \\
& $93039-02-02-03$ & $2008-11-3020: 18: 58.5$ & 15540 & & \\
& $93039-02-02-04$ & $2008-12-0102: 36: 03.2$ & 10310 & & \\
& $93039-02-02-05$ & $2008-12-0107: 18: 58.2$ & 5823 & & \\
\hline
\end{tabular}

Table 2. Log of AstroSat LAXPC observations used in this study.

\begin{tabular}{|c|c|c|c|c|}
\hline S. no. & Orbit & $\begin{array}{c}\text { Time of Observation } \\
\text { (yyyy-mm-dd hr:min:sec) }\end{array}$ & MJD (start) & Useful exposure (s) \\
\hline 1 & $\begin{array}{l}869 \\
870 \\
871 \\
872 \\
873 \\
874\end{array}$ & 2015-11-25 18:37:25-2015-11-26 04:02:47 & 57351.78 & 22052 \\
\hline 2 & $\begin{array}{l}18673 \\
18674 \\
18675 \\
18676 \\
18677 \\
18680\end{array}$ & 2019-03-12 16:31:52-2019-03-13 06:28:17 & 58554.68 & 34148 \\
\hline 3 & $\begin{array}{l}18716 \\
18717 \\
18718 \\
18719 \\
18720 \\
18721 \\
18723\end{array}$ & 2019-03-15 14:42:44-2019-03-16 03:30:05 & 58557.61 & 36697 \\
\hline
\end{tabular}

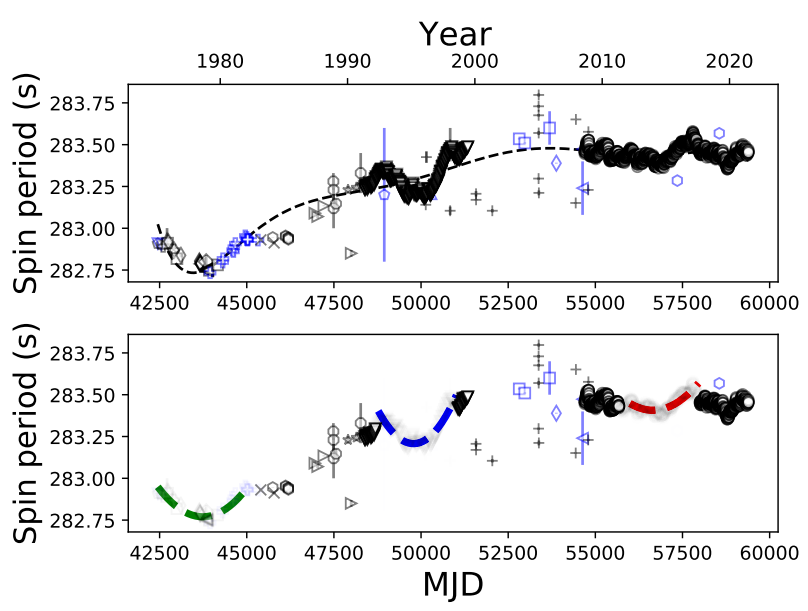

Figure 3. Long-term spin history of Vela X-1 from February, 1975 until June, 2021 (upper panel, same as shown in Fig. 2). Three distinct episodes of torque reversals from spin-up to spin-down occur around MJD 44000, 50000 and 57000 which have been fitted using a parabola shown in dashed green, blue and red curve respectively (bottom panel). The radius of curvatures of the parabolas fitted to the torque reversals around these epochs are different and show asymmetricity. of the neutron star, internal torques acting on the crust of the neutron star and/or magnetospheric state variations of the neutron star. We notice from Fig. 2 that the cyclic modulation in spin period is superposed on a long-term spin-down trend (starting around MJD 44000 until around MJD 53000). Fig. 5 shows the Lomb-Scargle periodogram of the long-term spin evolution of Vela X-1. We obtain the time-scale of nearly cyclic spin period evolution to be 2154.58 $\mathrm{d}\left(5.90_{-0.10}^{+0.05} \mathrm{yr}\right)$. Table 3 lists salient parameters of X-ray pulsars in which nearly cyclic spin period evolution on time-scales of years has been detected.

\section{DISCUSSIONS}

From the spin period measurements over a period of $46 \mathrm{yr}$, one can discern an overall trend of spin-down of the source though it will be noticed from Fig. 2 that since MJD 51000 the spin period has largely stayed at $\sim 283.4 \mathrm{~s}$ with erratic episodes of spin-up and spin-down. The results in the previous section show nearly periodic spin period variations in Vela X-1 on time-scales of about $5.9 \mathrm{yr}$ which is superposed on long-term spin-down trend since its discovery in 1975 . We also find nearly cyclic turnover from spin-up to spin-down regimes on decadal time-scales of about 17-19 yr (three cycles clearly identified in about $46 \mathrm{yr}$ of spin period evolution of this X-ray pulsar) which, to the best of our knowledge, has been detected in any accretion powered pulsar for the very first time. In 
Table 3. List of known X-ray pulsars which show nearly periodic spin period changes on long time-scales.

\begin{tabular}{cccccc}
\hline \hline Name of X-ray pulsar & Spin period (s) & Orbital period (d) & Spin change periodicity (yr) & wind/disc-fed & Reference \\
\hline Cen X-3 & $\sim 4.8$ & $\sim 2.1$ & $\sim 9.2$ & disk-fed & 1 \\
LMC X-4 & $\sim 13.5$ & $\sim 1.4$ & $\sim 6.8$ & disk-fed & 2 \\
Vela X-1 & $\sim 283$ & $\sim 8.9$ & $\sim 5.9$ & wind-fed & 3 (this work) \\
\hline
\end{tabular}

(1) Tsunemi (1989), (2) Molkov et al. (2016) and (3) this work.

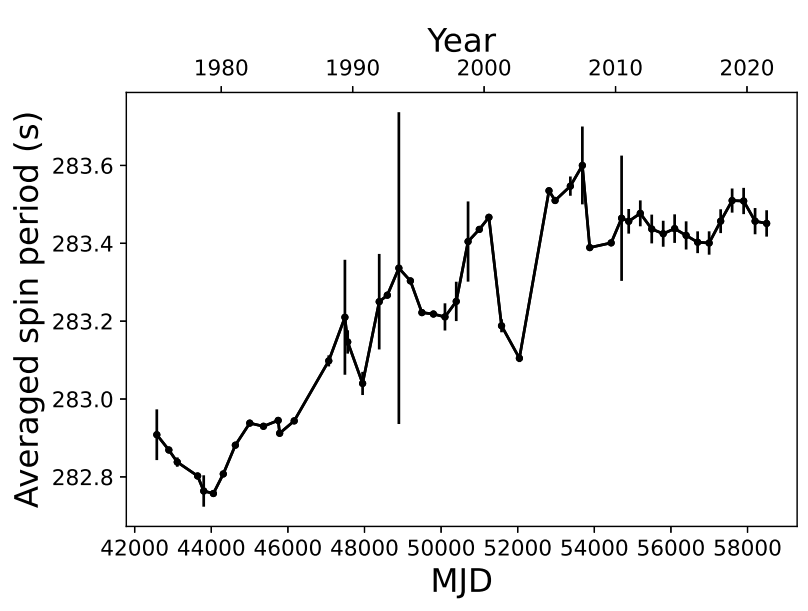

Figure 4. Long-term averaged spin history of Vela X-1 from 1975 February until 2021 June. The spin periods have been averaged using a time window of $300 \mathrm{~d}$. The presence of quasiperiodic episodes of spin-up and spin-down (on time-scales of about $2000 \mathrm{~d}$ ) superposed on long-term spin-down trend stands out.

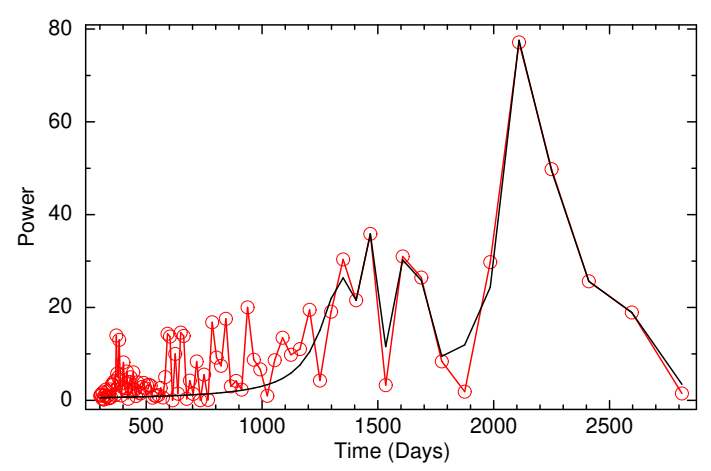

Figure 5. Lomb-Scargle periodogram of the long-term spin evolution of Vela X-1 shown earlier in Fig. 2. The periodogram peaks at $2154.58 \mathrm{~d}$ (about 5.9 $\mathrm{yr})$. The detected period is found to have more than $5 \sigma$ confidence.

the following section, we discuss possible mechanisms which can lead to nearly cyclic spin period variations on time-scales of years in wind-fed pulsars like Vela X-1. We would like to emphasize that as the temporal variation of accretion torques in wind-fed accretion powered pulsars is complex and depends on many parameters such as the stellar wind velocity, stellar wind density, magnetospheric state of the pulsar, energetics of the plasma flow in the vicinity of the neutron star etc. a combination of the following mechanisms may operate simultaneously in this wind-fed X-ray pulsar.

\subsection{Cyclic mass loss in the donor star HD 77581?}

Variations in the spin period of an accretion powered pulsar is the manifestation of the interplay of two torques acting on the compact object viz. the accretion torque exerted by the accreting matter captured from the stellar wind of the donor star (for a wind-fed pulsar such as Vela X-1) and the internal torque due to the dynamic coupling between the solid crust and the superfluid matter in the core of the neutron star (Nagase 1989). To decouple these two torques acting on a neutron star is a challenging exercise. It is believed that spin wandering on short time-scales can be caused either by variations in the accretion torque (Elsner \& Lamb 1976; Ghosh \& Lamb 1979) and/or by variations in the internal torque applied by the superfluid core (Lamb et al. 1978; Ghosh \& Lamb 1979) on the crust of the neutron star. It has also been suggested in some earlier studies that variations in spin period over sufficiently long time-scales is the manifestation of the external torque acting on the neutron star (Pringle \& Rees 1972; Lamb et al. 1973; Ghosh $\&$ Lamb 1979). Nagase et al. (1984b) suggest that the long-term (about 3 yr or longer) spin-down trend in Vela X-1 during 1979-81 is not caused due to the internal torque because it is less likely that the coupling between the crust and the superfluid core of the neutron star can sustain for such a long time. In addition, they also discuss about ineffectiveness of the internal torque in causing short-term, abrupt spin period changes. In the light of the above arguments, we surmise that the (external) accretion torque is the dominant torque acting on the neutron star over long time-scales (a few tens of years) and variations in this torque manifest as dynamic changes in the accretion torque and ultimately spin changes in Vela X-1 on long time-scales. Variations in this torque occur due to fluctuations in the accretion rate of matter from the stellar wind of the donor star. It is known that the stellar wind from early-type stars vary irregularly on time-scales from minutes to years and so it has been suggested that variations in the stellar wind from the donor star can cause reversals in the accretion torque acting on the neutron star on time-scales of days to years (Nagase 1989). Interestingly, Tsunemi (1989) surmise that the $9.2 \mathrm{yr}$ cyclic spin period variations detected in Cen X-3 maybe caused by changes in the activity of the massive O6-8 III companion star. Variations in the stellar activity of the companion star may lead to changes in the stellar wind velocity and density. This will result in varying mass loss rate which may modulate the accretion rate and hence correlate with spin period changes detected in Cen X-3 by Tsunemi (1989). Vela X-1 is a wind-fed pulsar accreting from an early-type star and therefore variations in mass-loss from the donor star will manifest as spin changes in the compact object on long time-scales. Thus, we infer that nearly periodic spin variations in Vela X-1 on long time-scales of about $5.9 \mathrm{yr}$ occur possibly due to cyclic 


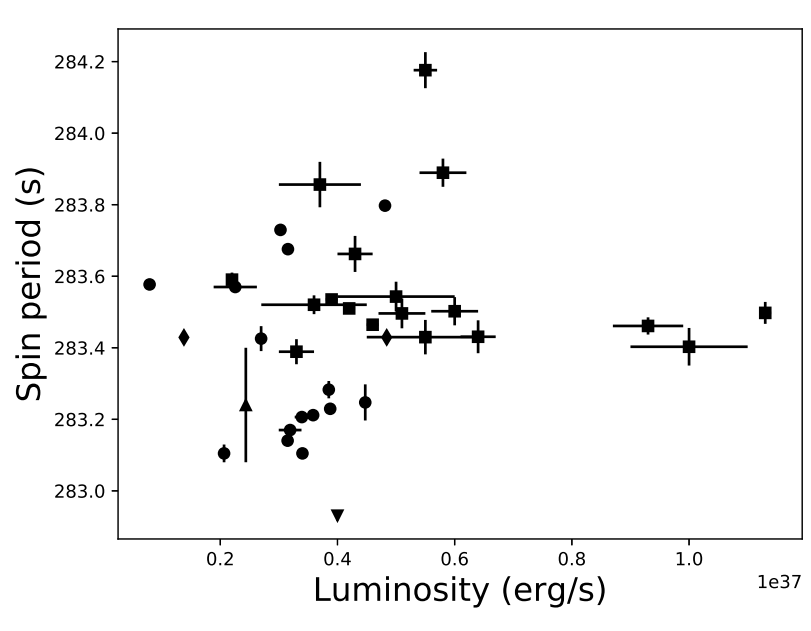

Figure 6. Plot showing spin period vs X-ray luminosity of Vela X-1. Different symbols indicate results obtained from our work and those available in the literature. Circles indicate luminosity in the range $2-80 \mathrm{keV}$ (this work), inverted triangle indicates luminosity in the range $2-30 \mathrm{keV}$ (Nagase et al. 1986), squares indicate luminosity in the range $3-100 \mathrm{keV}$ (Lutovinov \& Tsygankov 2009), triangle indicates luminosity in the range $0.3-70 \mathrm{keV}$ (Maitra \& Paul 2013) and diamonds indicate luminosity in the range 3-79 $\mathrm{keV}$ (Fuerst et al. 2013). Please note that energy ranges of luminosities are different and can be compared because the contribution to X-ray luminosity at higher energies decreases significantly.

mass-loss/stellar wind variations from the donor star HD 77581. Long-term optical monitoring of the companion star on decadal time-scales might shed light on this plausible mechanism.

Changes in X-ray luminosity of the compact object are a telltale signatures of variations in the accretion rate of captured matter from the stellar wind. We compare the luminosity and the pulse periods of Vela X-1 obtained using RXTE PCU2 data and those reported in literature (Fig. 6).

It should be noted that the range of energies for comparing luminosities are not identical but still we can compare them as the contribution of high energy photons to the X-ray luminosity, diminishes significantly with energy. We compute Pearson correlation coefficient for possible correlation between the spin period and X-ray luminosity and find it to be 0.2 indicating weak or no correlation between these parameters shown in Fig. 6. It has been observed that the luminosity and pulse period in Vela X-1 are not clearly correlated (Malacaria et al. 2020). However, some studies suggest that changes in the count rate on short time-scales can affect the spin of the neutron star on short time-scales of a few days (Kretschmar et al. 1997). Interestingly, a correlated behaviour between count rate in 11-40 keV energy range (not affected by photoelectric absorption) and spin period has been observed approximately over $50 \mathrm{~d}$ or so which suggests possible correlation between accretion torque and spin period on time-scales of about a couple of months (refer Table 1 in Kretschmar et al. (1997)). Lutovinov \& Tsygankov (2009) find using INTEGRAL observations that the luminosity of Vela X-1 enhances almost threefold during an outburst which is accompanied by remarkable changes in the spin period and suggests that episodic enhanced accretion during flares can cause tangible changes in the spin period. Interestingly, the source spectrum and the position of cyclotron lines did not vary during this period (Lutovinov \& Tsygankov 2009) which strongly suggests that the fluctuations in accretion rate was primarily responsible for sudden changes observed in the spin period during this period. However, these observed changes are detected at much shorter time-scales of about a few hours to few tens of days. Long-term X-ray monitoring of the pulsar at regular intervals using pointed mode instruments is required to investigate this premise in detail.

One can infer from Fig. 6 that there are variations in the pulsar period within the range $\delta \mathrm{P} / \mathrm{P} \sim 1 / 300$ while the corresponding $\mathrm{X}$ ray luminosity also changes by a factor of $\sim 3$ in the range $\sim$ $1.5-4 \times 10^{36} \mathrm{erg} \mathrm{s}^{-1}$. It has been shown by Shakura et al. (2012, 2014 , 2018) that the quasi-spherical settling accretion regime is feasible when the X-ray luminosity $\mathrm{L}_{X} \lesssim 4 \times 10^{36} \mathrm{erg} \mathrm{s}^{-1}$. At higher luminosities, the accretion proceeds in the supersonic Bondi regime. The following discussion is applicable for those states of the source when the $\mathrm{L}_{\mathrm{X}} \lesssim 4 \times 10^{36} \mathrm{erg} \mathrm{s}^{-1}$. The equilibrium period for quasispherical accretion is given by (Postnov et al. 2015)

$P_{e q}^{*} \sim 940[s] \mu_{30}^{12 / 11}\left(\frac{P_{b}}{10 d}\right) \dot{M}_{16}^{-4 / 11} v_{8}^{4}$,

where $\mu_{30}=\mu / 10^{30}\left[\mathrm{G} \mathrm{cm}^{3}\right]$ is the dipole magnetic moment given by $\mu=\mathrm{BR}^{3} / 2$ where $\mathrm{R}$ is the radius of the neutron star having typical value of $10 \mathrm{~km}, \dot{M}_{16}=\dot{M} / 10^{16}\left[\mathrm{~g} \mathrm{~s}^{-1}\right]$ is the accretion rate onto the neutron star, $P_{b}$ is the orbital period and $v_{8}=v / 10^{8}\left[\mathrm{~cm} \mathrm{~s}^{-1}\right]$ is the characteristic stellar wind velocity. Using equation 1 , we obtain

$\frac{\delta P}{P}=\frac{-4}{11} \frac{\delta L}{L}+4 \frac{\delta v}{v}$.

For the Bondi-Hoyle-Lyttleton wind accretion (Hoyle \& Lyttleton 1939; Bondi \& Hoyle 1944; Bondi 1952),

$\frac{\delta L}{L}=-4 \frac{\delta v}{v}+\frac{\delta \dot{M}_{o}}{\dot{M}_{o}}$,

where $\dot{M}_{o}$ is the wind mass-loss rate from the optical companion star. Using equation 2 and 3 we obtain,

$\frac{\delta P}{P}=\frac{-4}{11} \frac{\delta \dot{M}_{o}}{\dot{M}_{O}}+\frac{60}{11} \frac{\delta v}{v} \sim 3 \times 10^{-3}$.

Equation 4 suggests $\delta \dot{M}_{o} / \dot{M}_{o} \sim 15 \delta v / v$. Then from equation 2 and using $\delta L / L \sim 2\left(\delta L / L=2\left(L_{\max }-L_{\min }\right) /\left(L_{\max }+L_{\text {min }}\right)\right)$ for the X-ray luminosity excursion, we get $\delta v / v \sim 2 / 11$ which implies from equation $3, \delta \dot{M}_{o} / \dot{M}_{o} \sim 30 / 11 \sim 3$.

The mass-loss rate from the companion star has been estimated using different models and lie in the range of about $0.5 \times 10^{-6} \mathrm{M}_{\odot}$ $\mathrm{yr}^{-1}$ to $7 \times 10^{-6} \mathrm{M}_{\odot} \mathrm{yr}^{-1}$ (Lamers et al. 1976; Hutchings 1976; Conti 1978; Dupree et al. 1980; McCray et al. 1984; Sadakane et al. 1985; Sato et al. 1986; Prinja 1990; Sako et al. 1999; Van Loon et al. 2001; Watanabe et al. 2006; Krtička et al. 2012; Falanga et al. 2015; Manousakis \& Walter 2015b; Giménez-García et al. 2016; Sander et al. 2018). A detailed comparison of the massloss rates from the literature is given in Table 7 in Kretschmar et al. (2021). The mass-loss rate is estimated to within a factor of about 6 comparing recent estimates from literature (Krtička et al. 2012; Falanga et al. 2015; Manousakis \& Walter 2015b; GiménezGarcía et al. 2016; Sander et al. 2018). The fractional mass-loss rate variations $\left(\delta \dot{M} / \dot{M}=2\left(\dot{M}_{\max }-\dot{M}_{\min }\right) /\left(\dot{M}_{\max }+\dot{M}_{\min }\right)\right)$ estimated from literature is about 2 using $\dot{M}_{\text {min }}=0.5 \times 10^{-6} \mathrm{M}_{\odot} \mathrm{yr}^{-1}$ 
(Lamers et al. 1976) and $\dot{M}_{\max }=7 \times 10^{-6} \mathrm{M}_{\odot} \mathrm{yr}^{-1}$ (Hutchings 1976). Using recent estimates for $\dot{M}_{\text {min }}=0.65 \times 10^{-6} \mathrm{M}_{\odot} \mathrm{yr}^{-1}$ (Sander et al. 2018) and $\dot{M}_{\text {max }}=5.3 \times 10^{-6} \mathrm{M}_{\odot} \mathrm{yr}^{-1}$ (Falanga et al. 2015), we obtain estimated fractional wind mass-loss rate variability of about 2. It should be noted that different indirect methods of the mass-loss estimate have their own systematics, which are difficult to take into account. Interestingly, the estimated fractional wind massloss rate variability from observations (about a factor of 2 from recent estimates) agrees to within a factor of about 1.5 from the value estimated using equation 3 . This corroborates that the nearly periodic spin variations in Vela X-1 on time-scales of about $5.9 \mathrm{yr}$ occur most likely due to cyclic mass-loss from the companion star HD 77581.

\subsection{Switching magnetosphere model}

We explore another mechanism which can possibly result in torque reversals in the neutron star on long time-scales. It has been known for a long time that radio pulsars (which are usually isolated systems) show unexplained stochastic deviations in their spin-down behaviour (known as "timing noise") on varied time-scales of a few hundred days to a few tens of years. This manifestation is akin to the random-walk behaviour in spin frequency observed in wind-fed accretion powered pulsars like Vela X-1. In an interesting study of timing irregularities of a sample of 366 pulsars, Hobbs et al. (2010) found some radio pulsars showing quasiperiodic structures in their long-term timing residuals. From power spectrum analysis, significant periodicities ranging from about $1.4 \mathrm{yr}$ to $10 \mathrm{yr}$ were found in PSR B1540-06, PSR B1642-03, PSR B1818-04, PSR B1826-17, PSR B1828-11 and PSR B2148+63 (Hobbs et al. 2010). Interestingly, we also find quasiperiodic variations in the long-term spin evolution of Vela X-1 on time-scales of about $5.9 \mathrm{yr}$ which is comparable to those inferred for radio pulsars showing quasiperiodic changes in their timing residuals. The underlying phenomena causing quasiperiodic structures in timing noise of radio pulsars is elusive. However, it has been suggested that these changes are driven by changes in the magnetosphere of the neutron star (Lyne et al. 2010). In this "state-switching model", the magnetosphere of the neutron star is suggested to harbour two or more magnetospheric states which can be stable on time-scales of years but the pulsar can switch abruptly between these states driven by changes in the parameters regulating the spin-down (Lyne et al. 2010). This can possibly happen in Vela X-1 where the coupling between the dynamic magnetosphere and the neutron star can change in a quasiperiodic fashion. Interestingly, the disk-fed pulsar LMC X-4 has been found showing a near cyclic spin period evolution on time-scales of about $6.8 \mathrm{yr}$ (Molkov et al. 2016) which is within a factor of 1.2 of the inferred time-scale in wind-fed pulsar Vela X-1. Recent observations of transient pulsar V0332+53 suggests switching of coupling between the accretion disc and the neutron star magnetosphere in a disk-fed pulsar (Doroshenko et al. 2017).

\subsection{Episodic accretion from transient accretion disc in Vela $\mathrm{X}-1$ ?}

Vela X-1 is known to accrete from the dense, strong stellar wind from its supergiant companion star (typical mass loss rate of about $\dot{M} \sim 10^{-6} \mathrm{M}_{\odot} \mathrm{yr}^{-1}$ (Hutchings 1974; Dupree et al. 1980; Nagase et al. 1986; Sako et al. 1999)) which most likely does not fill its Roche lobe. However, Kretschmar et al. (2021) suggest that the mass transfer in Vela X-1 might happen due to both accretion from the stellar wind all throughout the orbit and frequent episodic Roche lobe overflow near the periastron passage. Possible observational signatures of intermittent Roche lobe overflow near periastron passage might be formation of short-lived transient accretion disc around the compact object which forms and dissipates on time-scales much lesser than the orbital period of the system and manifests as sudden spin-up/down of the neutron star showing correlation or anti-correlation between spin changes and X-ray luminosity. It is believed that transient accretion disks might fuel the sparse spin-up/spin-down episodes detected by the Fermi/GBM at irregular intervals (Malacaria et al. 2020). The spin-up rates observed during such events are an order of magnitude higher than the spin-down rates (Malacaria et al. 2020). However, conclusive signatures of formation of transient accretion disc in this system has evaded so far. The long-term impact of possible recurrent formation of transient accretion discs on the spin history of this pulsar is unclear. Earlier investigations of the spin evolution of the pulsar on shorter time-scales of a few days found random switches between spin-up and spin-down regimes, which is the well-known hallmark of wind-fed pulsars (Boynton et al. 1984, 1986; Deeter et al. 1989; Baykal \& Oegelman 1993; de Kool \& Anzer 1993; Bildsten et al. 1997). However, recent numerical studies suggest formation of temporary accretion discs in wind-fed X-ray pulsars (El Mellah et al. 2019b,a; Karino et al. 2019) but compelling evidence of their existence has been elusive. In a recent study, Liao et al. (2020) infer presence of temporary accretion disc in Vela X-1 during an extended low state lasting at least $30 \mathrm{ks}$ which was accompanied by an unusual spin-up event and similar Fe $\mathrm{K} \alpha$ fluxes compared to the preceding flaring period.

\subsection{Quasi-spherical accretion in Vela X-1}

The spin-up/spin-down variations in this pulsar can be explained using the theory of quasi-spherical accretion from the stellar wind of the companion star. The theory of quasi-spherical settling accretion has been discussed in detail by Shakura et al. (2012) and Shakura et al. (2014). According to this theory, two very different regimes of mass accretion onto the neutron star are possible depending on the X-ray luminosity of the pulsar. For high enough luminosity of about $4 \times 10^{36} \mathrm{erg} \mathrm{s}^{-1}$, the plasma in the bow shock cools rapidly by Compton process and so the matter falls freely (supersonically) toward the magnetosphere forming a shock in the vicinity of the magnetosphere. For lower luminosities, cooling is slightly quenched and hence the matter approaches the neutron star magnetosphere subsonically and forms a hot quasi-static shell around the magnetosphere which is referred to as the settling accretion. The accretion rate in this mode is determined by the ability of plasma to penetrate the magnetosphere via instabilities.

Vela X-1 observations satisfy the conditions for application of this model viz. slow spin period $\left(\mathrm{P}_{\mathrm{s}} \sim 283 \mathrm{~s}\right)$ and X-ray luminosity of about $4 \times 10^{36} \mathrm{erg} \mathrm{s}^{-1}$. The luminosity of Vela X-1 is about $4 \times 10^{36} \mathrm{erg} \mathrm{s}^{-1}$ (Kreykenbohm et al. 2002) and occasionally exceeds this threshold during flares and outbursts. The measured surface magnetic field of the pulsar is $\sim 2.7 \times 10^{12} \mathrm{G}$ (Kreykenbohm et al. 2002; Coburn et al. 2002) which is consistent with the expected equilibrium period of quasi-spherical accretion onto a neutron star in the regime of settling accretion (Postnov et al. 2015) given by equation 1.

In equation $1, P_{e q}^{*}$ depends strongly on the stellar wind velocity (proportional to the fourth power of the wind velocity). Assuming that the Vela $\mathrm{X}-1$ rotates about its equilibrium spin period and given that the surface magnetic field is measured using cyclotron 
resonance scattering features we can use it to estimate the stellar wind velocity of the matter captured by the neutron star.

$v_{8} \sim 0.57 \mu_{30}^{-3 / 11} \dot{M}_{16}^{1 / 11}\left(\frac{P_{e q}^{*} / 100 \mathrm{~s}}{P_{b} / 10 \mathrm{~d}}\right)^{1 / 4}$.

Using $\mu_{30}=1.35, \dot{M}_{16}=4.44$ (using $\mathrm{L}_{\mathrm{X}}=0.1 \dot{M} \mathrm{c}^{2}$ and $\left.\mathrm{L}_{\mathrm{X}}=4 \times 10^{36} \mathrm{erg} \mathrm{s}^{-1}\right), P_{e q}^{*}=283 \mathrm{~s}$ and $P_{b}=8.96 \mathrm{~d}$, we obtain $v_{8} \sim 0.8\left(v=800 \mathrm{~km} \mathrm{~s}^{-1}\right)$ which is approximately equal to that of $v=700 \mathrm{~km} \mathrm{~s}^{-1}$ inferred from observations (Giménez-García et al. 2016).

In the following discussion, we estimate the spin-up/spin-down rates from the quasi-settling accretion theory (Shakura et al. 2012, 2014, 2018) and compare it with the measured spin-up/spin-down rates. The estimated spin-up rate from the quasi-settling accretion theory is given by (Postnov et al. 2015)

$\dot{\omega}_{s u}^{*} \sim 10^{-9} \frac{\mathrm{Hz}}{\mathrm{d}} \Pi_{s u} v_{8}{ }^{-4} \mu_{30}^{1 / 11} \dot{M}_{16}^{7 / 11}\left(\frac{P_{b}}{10 \mathrm{~d}}\right)^{-1}$.

We obtain $\dot{\omega}_{s u}^{*} \sim 1.11 \times 10^{-7} \mathrm{~Hz} / \mathrm{d}$ using $\Pi_{s u} \sim 9$ (Shakura et al. 2012, 2014; Postnov et al. 2015; Shakura et al. 2018), $\dot{M}_{16}=$ 4.44, $\mu_{30}=1.35, v_{8} \sim 0.7$ and $P_{b}=8.96 \mathrm{~d}$.

The estimated spin-down rate from the quasi-settling accretion theory is given by (Postnov et al. 2015)

$\dot{\omega}_{s d}^{*} \sim 10^{-8} \frac{\mathrm{Hz}}{\mathrm{d}} \Pi_{s d} \mu_{30}^{13 / 11} \dot{M}_{16}^{3 / 11}\left(\frac{P^{*}}{100 \mathrm{~s}}\right)^{-1}$

We obtain $\dot{\omega}_{s d}^{*} \sim-6.8 \times 10^{-8} \mathrm{~Hz} / \mathrm{d}$ (using $\Pi_{s d} \sim 9$ (Shakura et al. 2012, 2014; Postnov et al. 2015; Shakura et al. 2018), $\dot{M}_{16}=$ 4.44, $\mu_{30}=1.35, P_{e q}^{*}=283 \mathrm{~s}$ and $P_{b}=8.96 \mathrm{~d}$ ) which is smaller than the deduced spin-up rate by a factor of about two. Fig. 7 shows the observed spin-up/down rates obtained from linear fit of BATSE and Fermi/GBM observations of this pulsar. It is clearly seen that the observed spin-up rate is usually higher than the spin-down rate. The average spin-up rate is $\sim 7.45 \times 10^{-8} \mathrm{~Hz} / \mathrm{d}$ which is a factor of 1.5 lower than that estimated using the quasi-settling accretion model. The average spin-down rate is $\sim-4.64 \times 10^{-8} \mathrm{~Hz} / \mathrm{d}$ which is also about a factor of 1.5 lower than that estimated using the quasisettling accretion model. Thus quasi-spherical accretion model can within a factor of $\sim 2$ reproduce the spin rates and may be the model that at least qualitatively explains the behaviour of Vela X-1.

\section{SUMMARY}

We have investigated the spin period evolution of Vela X-1 over a period of about five decades and detect the long-term spin-down apparition in this pulsar. We also find random, episodic spin changes on short time-scales superposed on the long-term spin-down manifestation. To our knowledge this is the first detection of spin evolution on such a long time-scale in a wind-fed pulsar. We have also detected periodic spin period variations in Vela X-1 on time-scales of about 5.9 yr. Our study might have useful ramifications for future explorations of long-term changes in accretion history/environment in other wind-fed X-ray pulsars and help to investigate and understand the underlying phenomena causing long-term nearly cyclic spin-changes in accretion powered pulsars.

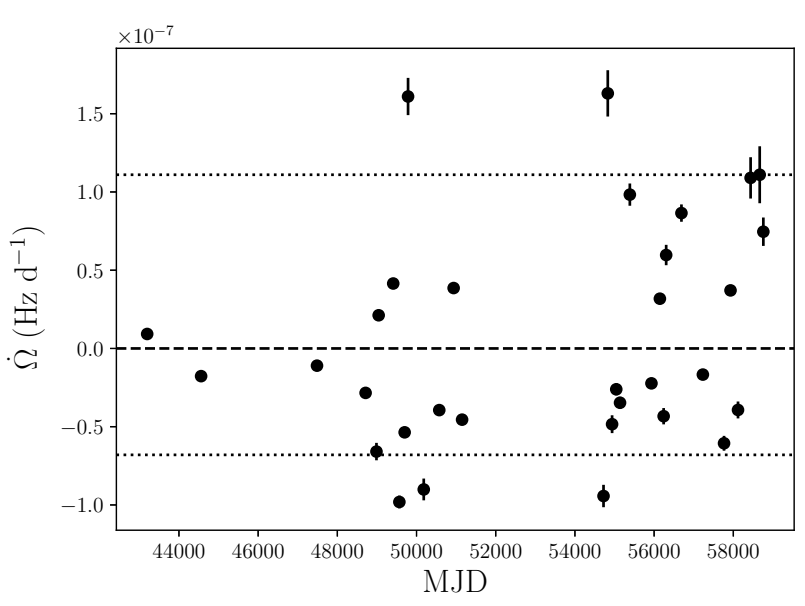

Figure 7. Plot showing estimated spin-up and spin-down rates spread over about four decades. The horizontal dotted lines show the estimated spinup and spin-down rates using the quasi-spherical settling accretion theory (Shakura et al. 2012, 2014).

\section{ACKNOWLEDGEMENTS}

This work is dedicated to the memory of Prof. Shashikumar Madhusudan Chitre, who sadly passed away on Monday 2021 January 11 . We are extremely thankful to the reviewer for carefully going through the manuscript and making detailed, valuable and constructive suggestions which have greatly improved the presentation of this paper. This research has made use of data obtained through the High Energy Astrophysics Science Archive Research Center (HEASARC) online service, provided by NASA/Goddard Space Flight Center. This publication uses the data from the AstroSat mission of the Indian Space Research Organisation (ISRO), archived at the Indian Space Science Data Centre (ISSDC). We thank members of LAXPC instrument team at TIFR and the AstroSat project team at URSC for their contributions to the development of the LAXPC instrument. We thank the LAXPC POC at TIFR for verifying and releasing the data. LAXPCSOFT software is used for analysis in this paper. This research has made use of software provided by the High Energy Astrophysics Science Archive Research Center (HEASARC), which is a service of the Astrophysics Science Division at NASA/GSFC. This research has also made use of the Fermi/GBM (Meegan et al. 2009) pulsar spin evolution history provided by the Fermi team. This research has also made use of the CGRO/BATSE pulsar spin evolution history provided by the BATSE team. This research has made use of NASA's Astrophysics Data System. ADC acknowledges support of the INSPIRE scholarship of the DST, Govt. of India as an undergraduate student and computing resources in the X-ray astronomy lab, UM-DAE CEBS. ADC acknowledges support of the INSPIRE fellowship of the DST, Govt. of India. JR acknowledges ISRO for funding support and IUCAA for their facilities.

\section{DATA AVAILABILITY}

This research has made use of archival data from the RXTE and the AstroSat mission. The CGRO/BATSE pulsar spin evolution history for Vela X-1 provided by the BATSE team is available at https://gammaray.nsstc.nasa.gov/batse/pulsar/data/ 
sources/velax1.html. The Fermi/GBM pulsar spin evolution history for Vela X-1 provided by the Fermi team is available at https://gammaray.nsstc.nasa.gov/gbm/science/ pulsars/lightcurves/velax1.html.

\section{REFERENCES}

Agrawal P., 2006, Advances in Space Research, 38, 2989

Agrawal P., et al., 2017, Journal of Astrophysics and Astronomy, 38, 30

Antia H., et al., 2017, The Astrophysical Journal Supplement Series, 231, 10

Bailer-Jones C., Rybizki J., Fouesneau M., Mantelet G., Andrae R., 2018, The Astronomical Journal, 156, 58

Barziv O., Kaper L., Van Kerkwijk M., Telting J., Van Paradijs J., 2001, Astronomy \& Astrophysics, 377, 925

Bautz M., Howe S., Gorecki A., Lang F., Levine A., Primini F., Lewin W., 1983, The Astrophysical Journal, 266, 794

Baykal A., Oegelman H., 1993, Astronomy and Astrophysics, 267, 119

Becker R., Rothschild R., Boldt E., Holt S., Pravdo S., Serlemitsos P., Swank J., 1978, The Astrophysical Journal, 221, 912

Bildsten L., et al., 1997, The Astrophysical Journal Supplement Series, 113, 367

Bondi H., 1952, Monthly Notices of the Royal Astronomical Society, 112, 195

Bondi H., Hoyle F., 1944, Monthly Notices of the Royal Astronomical Society, 104, 273

Boynton P., Deeter J., Lamb F., Zylstra G., Pravdo S., White N., Wood K., Yentis D., 1984, The Astrophysical Journal, 283, L53

Boynton P., Deeter J., Lamb F., Zylstra G., 1986, The Astrophysical Journal, 307,545

Bradt H., Rothschild R., Swank J., 1993, Astronomy and Astrophysics Supplement Series, 97, 355

Brucato R. J., Kristian J., 1972, The Astrophysical Journal, 173, L105

Camero-Arranz A., Finger M., Ikhsanov N., Wilson-Hodge C., Beklen E., 2009, The Astrophysical Journal, 708, 1500

Chakrabarty D., et al., 1993, Astrophysical Journal, 403, L33

Chakrabarty D., et al., 1997a, The Astrophysical Journal, 474, 414

Chakrabarty D., et al., 1997b, The Astrophysical Journal Letters, 481, L101

Chandra A. D., Roy J., Agrawal P., Choudhury M., 2020, Monthly Notices of the Royal Astronomical Society, 495, 2664

Charles P., Mason K., White N., Culhane J., Sanford P., Moffat A., 1978, Monthly Notices of the Royal Astronomical Society, 183, 813

Chodil G., Mark H., Rodrigues R., Seward F., Swift C., 1967, The Astrophysical Journal, 150, 57

Coburn W., Heindl W., Rothschild R., Gruber D., Kreykenbohm I., Wilms J., Kretschmar P., Staubert R., 2002, The Astrophysical Journal, 580, 394

Conti P., 1978, Astronomy and Astrophysics, 63, 225

Deeter J., Boynton P., Lamb F., Zylstra G., 1989, The Astrophysical Journal, 336, 376

Doroshenko V., Santangelo A., Suleimanov V., 2011, Astronomy \& Astrophysics, 529

Doroshenko V., Tsygankov S. S., Mushtukov A. A., Lutovinov A. A., Santangelo A., Suleimanov V. F., Poutanen J., 2017, Monthly Notices of the Royal Astronomical Society, 466, 2143

Ducci L., Sidoli L., Mereghetti S., Paizis A., Romano P., 2009, Monthly Notices of the Royal Astronomical Society, 398, 2152

Dupree A., et al., 1980, The Astrophysical Journal, 238, 969

El Mellah I., Sundqvist J., Keppens R., 2019a, Astronomy \& Astrophysics, $622, \mathrm{~L} 3$

El Mellah I., Sander A., Sundqvist J., Keppens R., 2019b, Astronomy \& Astrophysics, 622, A189

Elsner R., Lamb F., 1976, Nature, 262, 356

Falanga M., Bozzo E., Lutovinov A., Bonnet-Bidaud J., Fetisova Y., Puls J., 2015, Astronomy \& Astrophysics, 577, A130

Forman W., Jones C., Tananbaum H., Gursky H., Kellogg E., Giacconi R., 1973, The Astrophysical Journal, 182, L103
Fritz S., Kreykenbohm I., Wilms J., Staubert R., Bayazit F., Pottschmidt K., Rodriguez J., Santangelo A., 2006, Astronomy \& Astrophysics, 458, 885

Fuerst F., et al., 2013, The Astrophysical Journal, 780, 133

Fürst F., et al., 2010, Astronomy \& Astrophysics, 519, A37

Ghosh P., Lamb F., 1979, The Astrophysical Journal, 234, 296

Gilfanov M., et al., 1989, Soviet Astronomy Letters, 15, 291

Giménez-García A., et al., 2016, Astronomy \& Astrophysics, 591, A26

González-Galán A., Kuulkers E., Kretschmar P., Larsson S., Postnov K., Kochetkova A., Finger M., 2012, Astronomy \& Astrophysics, 537, A66

Haberl F., 1994, Astronomy and Astrophysics, 288, 791

Haberl F., White N., 1990, The Astrophysical Journal, 361, 225

Hiltner W., Werner J., Osmer P., 1972, The Astrophysical Journal, 175, L19

Hobbs G., Lyne A., Kramer M., 2010, Monthly Notices of the Royal Astronomical Society, 402, 1027

Hoyle F., Lyttleton R. A., 1939, in Mathematical Proceedings of the Cambridge Philosophical Society. pp 405-415

Hu C.-P., Ng C.-Y., Chou Y., 2016, Journal of Astronomy and Space Sciences, 33,173

Hutchings J., 1974, The Astrophysical Journal, 192, 685

Hutchings J., 1976, The Astrophysical Journal, 203, 438

Inam S., Şahiner Ş., Baykal A., 2009, Monthly Notices of the Royal Astronomical Society, 395, 1015

Inoue H., Ogawara Y., Ohashi T., Waki I., Hayakawa S., Kunieda H., Nagase F., Tsunemi H., 1984, Publications of the Astronomical Society of Japan, 36,709

Jahoda K., et al., 1996, in SPIE Proc. 2808, ed. OHV Sigmund \& M. Gumm (Bellingham: SPIE).

Ji L., Staubert R., Ducci L., Santangelo A., Zhang S., Chang Z., 2019, Monthly Notices of the Royal Astronomical Society, 484, 3797

Jones C., Liller W., 1973, The Astrophysical Journal, 184, L121

Joss P. C., Rappaport S. A., 1984, Annual review of astronomy and astrophysics, 22, 537

Karino S., Nakamura K., Taani A., 2019, Publications of the Astronomical Society of Japan, 71, 58

Kelley R., Jernigan J., Levine A., Petro L., Rappaport S., 1983, The Astrophysical Journal, 264, 568

Kretschmar P., et al., 1996, Astronomy and Astrophysics Supplement Series, 120,175

Kretschmar P., et al., 1997, Astronomy and Astrophysics, 325, 623

Kretschmar P., et al., 2021, arXiv preprint arXiv:2104.13148

Kreykenbohm I., Kretschmar P., Wilms J., Staubert R., Kendziorra E., Gruber D., Heindl W., Rothschild R., 1999, Astronomy and Astrophysics, 341,141

Kreykenbohm I., Coburn W., Wilms J., Kretschmar P., Staubert R., Heindl W. A., Rothschild R. E., 2002, Astronomy \& Astrophysics, 395, 129

Kreykenbohm I., et al., 2008, Astronomy \& Astrophysics, 492, 511

Krtička J., Kubát J., Skalickỳ J., 2012, The Astrophysical Journal, 757, 162

Krzeminski W., 1974, The Astrophysical Journal, 192, L135

La Parola V., Cusumano G., Segreto A., D'Aì A., 2016, Monthly Notices of the Royal Astronomical Society, 463, 185

Lamb F., Pethick C., Pines D., 1973, The Astrophysical Journal, 184, 271

Lamb F., Pines D., Shaham J., 1978, The Astrophysical Journal, 224, 969

Lamers H., Van den Heuvel E., Petterson J., 1976, Astronomy and Astrophysics, 49,327

Lang F., et al., 1981, The Astrophysical Journal, 246, L21

Lapshov I. Y., Syunyaev R., Chichkov M., Dremin V., Brandt S., Lund N., 1992, Soviet Astronomy Letters, 18, 16

Levine A. M., Bradt H., Cui W., Jernigan J., Morgan E. H., Remillard R., Shirey R. E., Smith D. A., 1996, The Astrophysical Journal Letters, 469, L33

Liao Z., Liu J., Zheng X., Gou L., 2020, Monthly Notices of the Royal Astronomical Society, 492, 5922

Lutovinov A., Tsygankov S., 2009, Astronomy Letters, 35, 433

Lutovinov A., Grebenev S., Syunyaev R., Pavlinskii M., 1994, Astronomy Letters, 20, 538

Lyne A., Hobbs G., Kramer M., Stairs I., Stappers B., 2010, Science, 329, 408 
Maitra C., Paul B., 2013, The Astrophysical Journal, 763, 79

Makishima K., et al., 1988, Nature, 333, 746

Makishima K., Mihara T., Nagase F., Tanaka Y., 1999, The Astrophysical Journal, 525, 978

Malacaria C., Jenke P., Roberts O., Wilson-Hodge C., Cleveland W., Mailyan B., et al., 2020, The Astrophysical Journal, 896, 90

Manousakis A., Walter R., 2015a, Astronomy \& Astrophysics, 575, A58

Manousakis A., Walter R., 2015b, Astronomy \& Astrophysics, 584, A25

Martínez-Núñez S., et al., 2014, Astronomy \& Astrophysics, 563, A70

McClintock J., et al., 1976, The Astrophysical Journal, 206, L99

McCray R., Kallman T. R., Castor J. I., Olson G. L., 1984, The Astrophysical Journal, 282, 245

Meegan C., et al., 2009, The Astrophysical Journal, 702, 791

Molkov S., Lutovinov A., Falanga M., Tsygankov S., Bozzo E., 2016, Monthly Notices of the Royal Astronomical Society, 464, 2039

Nagase F., 1989, Publications of the Astronomical Society of Japan, 41, 1

Nagase F., et al., 1981, Nature, 290, 572

Nagase F., Hayakawa S., Makino F., Sato N., Makishima K., 1983, Publications of the Astronomical Society of Japan, 35, 47

Nagase F., et al., 1984a, Publications of the Astronomical Society of Japan, 36,667

Nagase F., et al., 1984b, The Astrophysical Journal, 280, 259

Nagase F., Hayakawa S., Sato N., Masai K., Inoue H., 1986, Publications of the Astronomical Society of Japan, 38, 547

Odaka H., Khangulyan D., Tanaka Y. T., Watanabe S., Takahashi T., Makishima K., 2013, The Astrophysical Journal, 767, 70

Orlandini M., et al., 1997, in AIP Conference Proceedings. pp 793-797

Postnov K., Mironov A., Lutovinov A., Shakura N., Kochetkova A. Y., Tsygankov S., 2015, Monthly Notices of the Royal Astronomical Society, 446, 1013

Pringle J., Rees M., 1972, Astronomy and Astrophysics, 21, 1

Prinja R., 1990, Monthly Notices of the Royal Astronomical Society, 246, 392

Quaintrell H., Norton A. J., Ash T., Roche P., Willems B., Bedding T., Baldry I., Fender R. P., 2003, Astronomy \& Astrophysics, 401, 313

Rappaport S., Joss P., 1977, Nature, 266, 683

Rappaport S., McClintock J., 1975, Discovery of a 283 second period in Vela X-1

Rappaport S., Joss P., McClintock J., 1976, The Astrophysical Journal, 206, L103

Rappaport S., Joss P., Stothers R., 1980, The Astrophysical Journal, 235, 570

Raubenheimer B., 1990, Astronomy and Astrophysics, 234, 172

Raubenheimer B., Oegelman H., 1990, Astronomy and Astrophysics, 230, 73

Rawls M. L., Orosz J. A., McClintock J. E., Torres M. A., Bailyn C. D., Buxton M. M., 2011, The Astrophysical Journal, 730, 25

Rothschild R., et al., 1998, The Astrophysical Journal, 496, 538

Roy J., et al., 2016, Experimental Astronomy, 42, 249

Roy J., et al., 2019, The Astrophysical Journal, 872, 33

Sadakane K., Hirata R., Jugaku J., Kondo Y., Matsuoka M., Tanaka Y., Hammerschlag-Hensberge G., 1985, The Astrophysical Journal, 288, 284

Sako M., Liedahl D. A., Kahn S. M., Paerels F., 1999, The Astrophysical Journal, 525, 921

Sander A. A., Fürst F., Kretschmar P., Oskinova L. M., Todt H., Hainich R., Shenar T., Hamann W.-R., 2018, Astronomy \& Astrophysics, 610, A60

Sato N., et al., 1986, Publications of the Astronomical Society of Japan, 38, 731

Scargle J. D., 1982, The Astrophysical Journal, 263, 835

Schanne S., Götz D., Gérard L., Sizun P., Falanga M., Hamadache C., Cordier B., von Kienlin A., 2007, in The Obscured Universe. Proceedings of the VI INTEGRAL Workshop. p. 479

Shakura N., Postnov K., Kochetkova A., Hjalmarsdotter L., 2012, Monthly Notices of the Royal Astronomical Society, 420, 216

Shakura N., Postnov K., Hjalmarsdotter L., 2013, Monthly Notices of the Royal Astronomical Society, 428, 670
Shakura N., Postnov K., Kochetkova A. Y., Hjalmarsdotter L., 2014, in EPJ Web of Conferences. p. 02001

Shakura N., Postnov K., Kochetkova A., Hjalmarsdotter L., 2018, in , Accretion Flows in Astrophysics. Springer, pp 331-392

Sidoli L., Paizis A., Fürst F., Torrejon J. M., Kretschmar P., Bozzo E., Pottschmidt K., 2015, Monthly Notices of the Royal Astronomical Society, 447, 1299

Staubert R., Kendziorra E., Pietsch W., Reppin C., Trümper J., Voges W., 1980, The Astrophysical Journal, 239, 1010

Staubert R., et al., 2004, in 5th INTEGRAL Workshop on the INTEGRAL Universe. p. 259

Taam R. E., Fryxell B., 1989, The Astrophysical Journal, 339, 297

Tjemkes S., Zuiderwijk E., Van Paradijs J., 1986, Astronomy and Astrophysics, 154,77

Tsunemi H., 1989, Publications of the Astronomical Society of Japan, 41, 453

Tsunemi H., Kitamoto S., Manabe M., Miyamoto S., Yamashita K., Nakagawa M., 1989, Publications of the Astronomical Society of Japan, 41, 391

Van Loon J. T., Kaper L., Hammerschlag-Hensberge G., 2001, Astronomy \& Astrophysics, 375, 498

Van Paradijs J., Hammerschlag-Hensberge G., Van den Heuvel E., Takens R., Zuiderwijk E., De Loore C., 1976, Nature, 259, 547

Vasilopoulos G., Petropoulou M., Koliopanos F., Ray P., Bailyn C., Haberl F., Gendreau K., 2019, arXiv preprint arXiv:1905.03740

Vidal N., Wickramasinghe D., Peterson B., 1973, The Astrophysical Journal, 182, L77

Watanabe S., et al., 2006, The Astrophysical Journal, 651, 421

Watson M., Griffiths R., 1977, Monthly Notices of the Royal Astronomical Society, 178, 513

Wilson R., Fishman G., Finger M., Pendleton G., Prince T., Chakrabarty D., 1993, in AIP Conference Proceedings. pp 291-302

Yadav J., et al., 2016, in Space Telescopes and Instrumentation 2016: Ultraviolet to Gamma Ray. p. 99051D

Ziolkowski J., 1985, Acta astronomica, 35, 185

de Kool M., Anzer U., 1993, Monthly Notices of the Royal Astronomical Society, 262, 726

van Kerkwijk M., van Paradijs J., Zuiderwijk E., Hammerschlag-Hensberge G., Kaper L., Sterken C., 1995, Astronomy and Astrophysics, 303, 483 van der Klis M., Bonnet-Bidaud J., 1984, Astronomy and Astrophysics, 135, 155 
Table 1: Log of all Vela X-1 pulse-period measurements used in the present work. Pulse period measurements from Fermi/GBM and CGRO/BATSE are publicly available at https://gammaray.msfc.nasa.gov/gbm/science/pulsars/ lightcurves/velax1.html and https://gammaray.msfc.nasa.gov/ batse/pulsar/data/sources/velax1.html respectively.

\begin{tabular}{|c|c|c|c|c|c|c|}
\hline Year & Month & MJD & Spin period (s) & Uncertainty (s) & Satellite & Reference \\
\hline 1975 & Feb & 42448.00 & 282.90830 & 0.00340 & Copernicus & Charles et al. (1978) \\
\hline 1975 & Feb & 42449.30 & 282.90830 & 0.00120 & $O A O 3$ & Charles et al. (1978) \\
\hline 1975 & May & 42551.00 & 282.90100 & 0.01100 & Copernicus & Charles et al. (1978) \\
\hline 1975 & May & 42552.30 & 282.90100 & 0.00370 & $\mathrm{OAO} 3$ & Charles et al. (1978) \\
\hline 1975 & Jul & 42600.60 & 282.89160 & 0.00040 & $S A S 3$ & Rappaport et al. (1976) \\
\hline 1975 & Oct & 42713.50 & 282.93700 & 0.06400 & Ariel V & Charles et al. (1978) \\
\hline 1975 & Nov & 42728.40 & 282.91080 & 0.00120 & $\operatorname{COS} B$ & van der Klis \& Bonnet-Bidaud (1984) \\
\hline 1975 & Dec & 42750.30 & 282.91900 & 0.00300 & OSO 8 & Becker et al. (1978) \\
\hline 1976 & May & 42900.00 & 282.87000 & 0.00400 & SAS 3 & Rappaport \& Joss (1977) \\
\hline 1976 & May & 42920.00 & 282.86900 & 0.00300 & OSO 8 & Becker et al. (1978) \\
\hline 1976 & Aug & 42997.10 & 282.81830 & 0.00030 & $\cos B$ & van der Klis \& Bonnet-Bidaud (1984) \\
\hline 1976 & Nov & 43112.00 & 282.83800 & 0.01200 & OSO 8 & Becker et al. (1978) \\
\hline 1978 & May & 43640.01 & 282.80452 & 0.00169 & OSO 8 & Deeter et al. (1989) \\
\hline 1978 & May & 43645.62 & 282.80016 & 0.00127 & OSO 8 & Deeter et al. (1989) \\
\hline 1978 & May & 43651.04 & 282.79188 & 0.00088 & OSO 8 & Deeter et al. (1989) \\
\hline 1978 & May & 43654.00 & 282.78700 & 0.00400 & HEAO 1 & Bautz et al. (1983) \\
\hline 1978 & May & 43656.02 & 282.78806 & 0.00127 & OSO 8 & Deeter et al. (1989) \\
\hline 1978 & May & 43660.75 & 282.78884 & 0.00099 & OSO 8 & Deeter et al. (1989) \\
\hline 1978 & May & 43665.47 & 282.78899 & 0.00127 & OSO 8 & Deeter et al. (1989) \\
\hline 1978 & May & 43669.99 & 282.79104 & 0.00110 & OSO 8 & Deeter et al. (1989) \\
\hline 1978 & Nov & 43824.50 & 282.74860 & 0.00040 & $S A S 3$ & Rappaport et al. (1980) \\
\hline 1978 & Nov & 43825.78 & 282.74884 & 0.00005 & HEAO 1 & Deeter et al. (1989) \\
\hline 1978 & Nov & 43836.80 & 282.80000 & 0.04000 & AIT/MPI balloon X-ray detector & Staubert et al. (1980) \\
\hline 1978 & Nov & 43840.43 & 282.74606 & 0.00027 & HEAO 1 & Deeter et al. (1989) \\
\hline 1978 & Dec & 43846.77 & 282.75233 & 0.00020 & HEAO 1 & Deeter et al. (1989) \\
\hline 1978 & Dec & 43850.00 & 282.75130 & 0.00050 & HEAO 1 & Bautz et al. (1983) \\
\hline 1978 & Dec & 43853.10 & 282.75604 & 0.00019 & HEAO 1 & Deeter et al. (1989) \\
\hline 1978 & Dec & 43857.59 & 282.75640 & 0.00037 & HEAO 1 & Deeter et al. (1989) \\
\hline 1978 & Dec & 43859.94 & 282.75881 & 0.00087 & HEAO 1 & Deeter et al. (1989) \\
\hline 1978 & Dec & 43861.38 & 282.75240 & 0.00108 & HEAO 1 & Deeter et al. (1989) \\
\hline 1978 & Dec & 43862.75 & 282.74948 & 0.00094 & HEAO 1 & Deeter et al. (1989) \\
\hline 1978 & Dec & 43864.21 & 282.75004 & 0.00068 & HEAO 1 & Deeter et al. (1989) \\
\hline 1978 & Dec & 43866.59 & 282.74616 & 0.00029 & HEAO 1 & Deeter et al. (1989) \\
\hline 1978 & Dec & 43871.00 & 282.75346 & 0.00021 & HEAO 1 & Deeter et al. (1989) \\
\hline 1979 & Jan & 43881.64 & 282.74632 & 0.00014 & HEAO 1 & Deeter et al. (1989) \\
\hline 1979 & Jan & 43891.30 & 282.75020 & 0.00088 & HEAO 1 & Deeter et al. (1989) \\
\hline 1979 & Mar & 43948.10 & 282.73370 & 0.00090 & Hakucho & Nagase et al. (1984b) \\
\hline 1979 & Oct & 44161.40 & 282.78090 & 0.00030 & $\operatorname{COS} B$ & van der Klis \& Bonnet-Bidaud (1984) \\
\hline 1980 & Mar & 44308.70 & 282.79770 & 0.00060 & Hakucho & Nagase et al. (1984b) \\
\hline 1980 & Mar & 44318.10 & 282.81740 & 0.00070 & Hakucho & Nagase et al. (1984b) \\
\hline 1980 & Dec & 44594.90 & 282.86930 & 0.00060 & Hakucho & Nagase et al. (1984b) \\
\hline 1981 & Jan & 44617.90 & 282.86080 & 0.00050 & Hakucho & Nagase et al. (1984b) \\
\hline 1981 & Jan & 44629.30 & 282.88720 & 0.00060 & Hakucho & Nagase et al. (1984b) \\
\hline 1981 & Mar & 44672.40 & 282.90850 & 0.00040 & Hakucho & Nagase et al. (1984b) \\
\hline 1981 & Dec & 44958.60 & 282.95450 & 0.00100 & Hakucho & Nagase et al. (1984b) \\
\hline 1982 & Jan & 44993.80 & 282.94510 & 0.00070 & Hakucho & Nagase et al. (1984b) \\
\hline 1982 & Jan & 45004.60 & 282.93150 & 0.00100 & Hakucho & Nagase et al. (1984b) \\
\hline 1982 & Feb & 45014.00 & 282.93500 & 0.00130 & Hakucho & Nagase et al. (1984b) \\
\hline 1982 & $\mathrm{Feb}$ & 45023.50 & 282.92870 & 0.00220 & Hakucho & Nagase et al. (1984b) \\
\hline 1982 & Mar & 45031.80 & 282.93370 & 0.00120 & Hakucho & Nagase et al. (1984b) \\
\hline 1982 & Dec & 45320.80 & 282.92930 & 0.00050 & Hakucho & Nagase et al. (1984b) \\
\hline 1983 & Mar & 45404.00 & 282.93060 & 0.00030 & Tenma & Nagase et al. (1984a) \\
\hline
\end{tabular}


Table 1 - Continued from previous page

\begin{tabular}{|c|c|c|c|c|c|c|}
\hline Year & Month & MJD & Spin period (s) & Uncertainty (s) & Satellite & Reference \\
\hline 1984 & Feb & 45746.29 & 282.94500 & 0.0009 & EXOSAT & Raubenheimer \& Oegelman (1990) \\
\hline 1984 & Mar & 45785.00 & 282.91200 & 0.00500 & Tenma & Sato et al. (1986) \\
\hline 1985 & Feb & 46110.06 & 282.94940 & 0.0002 & EXOSAT & Raubenheimer \& Oegelman (1990) \\
\hline 1985 & $\mathrm{Feb}$ & 46113.96 & 282.95490 & 0.0001 & EXOSAT & Raubenheimer \& Oegelman (1990) \\
\hline 1985 & May & 46186.07 & 282.94330 & 0.0005 & EXOSAT & Raubenheimer \& Oegelman (1990) \\
\hline 1985 & May & 46191.14 & 282.94410 & 0.0007 & EXOSAT & Raubenheimer \& Oegelman (1990) \\
\hline 1985 & May & 46193.06 & 282.93830 & 0.0003 & EXOSAT & Raubenheimer \& Oegelman (1990) \\
\hline 1985 & May & 46194.97 & 282.9331 & 0.0008 & EXOSAT & Raubenheimer \& Oegelman (1990) \\
\hline 1987 & Jun & 46954.00 & 283.09000 & 0.01000 & Ginga & Tsunemi et al. (1989) \\
\hline 1987 & Aug & 47035.00 & 283.07000 & 0.01000 & Ginga & Tsunemi (1989) \\
\hline 1988 & $\mathrm{Feb}$ & 47211.50 & 283.13400 & 0.00200 & Ginga & Nagase (1989) \\
\hline 1988 & Nov & 47485.00 & 283.28000 & 0.05000 & KVANT & Kretschmar et al. (1997) \\
\hline 1988 & Nov & 47490.00 & 283.23000 & 0.07000 & KVANT & Kretschmar et al. (1997) \\
\hline 1988 & Nov & 47491.00 & 283.12000 & 0.12000 & KVANT & Kretschmar et al. (1997) \\
\hline 1989 & $\mathrm{Feb}$ & 47561.00 & 283.1466 & 0.03000 & $K V A N T$ & Gilfanov et al. (1989) \\
\hline 1990 & Jan & 47906.00 & 283.23 & 0.022 & GRANAT & Lapshov et al. (1992) \\
\hline 1990 & Apr & 47995.00 & 282.85 & 0.02 & Ginga & Makishima et al. (1999) \\
\hline 1990 & Sept & 48149.00 & 283.244 & 0.022 & GRANAT & Lapshov et al. (1992) \\
\hline 1991 & Jan & 48269.00 & 283.33000 & 0.12000 & KVANT & Kretschmar et al. (1997) \\
\hline 1991 & Feb & 48302.00 & 283.26 & 0.013 & GRANAT & Lapshov et al. (1992) \\
\hline 1992 & Jun & 48787.00 & 283.32600 & 0.02000 & GRANAT & Lutovinov et al. (1994) \\
\hline 1992 & Nov & 48945.00 & 283.20000 & 0.40000 & $R O S A T$ & Haberl (1994) \\
\hline 1996 & $\mathrm{Feb}$ & 50135.16 & 283.14032 & 0.00168 & $R X T E$ & present work \\
\hline 1996 & Mar & 50153.74 & 283.42560 & 0.03488 & $R X T E$ & present work \\
\hline 1996 & Jul & 50288.00 & 283.20600 & 0.00100 & BepposAX & Orlandini et al. (1997) \\
\hline 1996 & Jul & 50291.86 & 283.24722 & 0.05048 & RXTE & present work \\
\hline 1996 & Aug & 50316.87 & 283.2185 & 0.0018 & CGRO/BATSE & Bildsten et al. (1997) \\
\hline 1998 & Jan & 50834.98 & 283.10471 & 0.02481 & RXTE & present work \\
\hline 1998 & Jan & 50835.65 & 283.5 & 0.1 & $R X T E$ & Kreykenbohm et al. (2002) \\
\hline 2000 & Feb & 51577.30 & 283.20627 & 0.01624 & $R X T E$ & present work \\
\hline 2000 & $\mathrm{Feb}$ & 51578.33 & 283.16998 & 0.00378 & $R X T E$ & present work \\
\hline 2001 & May & 52044.12 & 283.10471 & 0.00980 & $R X T E$ & present work \\
\hline 2003 & Jun & 52816.50 & 283.53500 & 0.00500 & INTEGRAL & Staubert et al. (2004) \\
\hline 2003 & Nov & 52976.00 & 283.51000 & 0.00500 & INTEGRAL & Staubert et al. (2004) \\
\hline 2005 & Jan & 53371.22 & 283.29715 & 0.00589 & RXTE & present work \\
\hline 2005 & Jan & 53372.50 & 283.72955 & 0.00827 & $R X T E$ & present work \\
\hline 2005 & Jan & 53373.52 & 283.67569 & 0.00742 & $R X T E$ & present work \\
\hline 2005 & Jan & 53374.54 & 283.56989 & 0.01233 & RXTE & present work \\
\hline 2005 & Jan & 53375.54 & 283.79736 & 0.00958 & $R X T E$ & present work \\
\hline 2005 & Jan & 53379.29 & 283.21161 & 0.01456 & $R X T E$ & present work \\
\hline 2005 & Nov & 53688.00 & 283.60000 & 0.10000 & INTEGRAL & Schanne et al. (2007) \\
\hline 2006 & May & 53881.16 & 283.389 & 0.004 & XMM-Newton & Martínez-Núñez et al. (2014) \\
\hline 2007 & Dec & 54437.39 & 283.15128 & 0.00308 & $R X T E$ & present work \\
\hline 2007 & Dec & 54442.42 & 283.65094 & 0.00003 & $R X T E$ & present work \\
\hline 2008 & Jun & 54634.00 & 283.473 & 0.004 & Suzaku & Doroshenko et al. (2011) \\
\hline 2008 & Jun & 54634.00 & 283.24000 & 0.16000 & Suzaku & Maitra \& Paul (2013) \\
\hline 2008 & Nov & 54795.52 & 283.22940 & 0.00674 & $R X T E$ & present work \\
\hline 2008 & Dec & 54801.04 & 283.57703 & 0.00452 & $R X T E$ & present work \\
\hline 2012 & Jul & 56117.78 & 283.429 & 0.0024 & NuSTAR & Fuerst et al. (2013) \\
\hline 2013 & Apr & 56405.04 & 283.429 & 0.0006 & NuSTAR & Fuerst et al. (2013) \\
\hline 2015 & Nov & 57351.78 & 283.28504 & 0.00001 & AstroSat & present work \\
\hline 2019 & Mar & 58554.68 & 283.56792 & 0.00001 & AstroSat & present work \\
\hline 2019 & Mar & 58557.61 & 283.61553 & 0.00001 & AstroSat & present work \\
\hline
\end{tabular}


This paper has been typeset from a $\mathrm{TEX}_{\mathrm{X}} / \mathrm{L} \mathrm{TEX}$ file prepared by the author. 